\title{
Evaluation of root morphology and root canal configuration of premolars in the Turkish individuals using cone beam computed tomography
}

\author{
Duygu Goller Bulut ${ }^{1}$, Emre Kose ${ }^{1}$, Gozde Ozcan ${ }^{1}$, Ahmet Ercan Sekerci $^{1}$, \\ Emin Murat Canger ${ }^{1}$, Yıldıray Sisman ${ }^{1}$
}

Correspondence: Dr. Gozde Ozcan

Email: gozcan89@gmail.com

\author{
'Department of Oral and Maxillofacial Radiology, \\ University of Erciyes, Kayseri, Turkiye
}

\section{ABSTRACT}

Objective: The aim of the present study is to assess the root and root canal morphology of maxillary and mandibular premolars in a Turkish population by using cone beam computed tomography (CBCT). Materials and Methods: In this study, CBCT images of 2134 premolars (987 maxillary, 1147 mandibular) were obtained from 404 patients. Details of gender, age, number of roots and canals, and canal configuration in each root were recorded. The canal configuration was classified and evaluated according to Vertucci's criteria. Results: The majority of maxillary premolars had two separate roots; although, three roots were identified in $1 \%$ of maxillary first premolars. However, most of the mandibular premolars had a single root. The two canals $(69.9 \%)$ and type I (62.6\%) and type II (34.1\%) configuration for upper first premolar, one canal (82.1\%) and type I (77.6\%) canal configuration for second premolar was the most prevalent root canal frequency. The most prevalent root canal frequency was the one canal (96.2\%) and type I (94.2\%) and type V (3.2\%) configuration for mandibular first premolar, one canal (98.9\%) and type I $(98.9 \%)$ canal configuration for second premolar. There was no difference in the root canal configurations and the numbers of canals between the left and the right side of both females and males $(P>0.05)$. Conclusions: Recognition of morphology and anatomy of the root canal system is one of the most important factors for successful endodontic treatment. Preoperative CBCT examination allows determination of root canal configuration of premolar teeth and helps clinicians in root canal treatment.

Key words: Cone beam computed tomography, mandibular premolars, maxillary premolars, root canal anatomy, root canal morphology

\section{INTRODUCTION}

Successful endodontic treatment depends on the clear understanding of configurations and shapes of root canals and the dimensions of the canal walls. ${ }^{[1]}$ Nevertheless, the variation of the root canal morphology presents clinical difficulties that often might lead to unfavorable endodontical outcomes. ${ }^{[2]}$ Radiographic investigation is important in diagnosis and root canal therapy planning in endodontics. The information obtained from digital periapical radiographs and conventional radiographs is limited due to three-dimensional (3-D) anatomy of the region being radiographed is compacted into a two-dimensional (2-D) image and this caused superimposition on buccolingual plane. Therefore, variableness of root and root canal morphology cannot be determined without 3-D images. ${ }^{[3]}$

Cone beam computed tomography (CBCT) is a noninvasive method, potentially provides the clinician

This is an open access article distributed under the terms of the Creative Commons Attribution-NonCommercial-ShareAlike 3.0 License, which allows others to remix, tweak, and build upon the work non-commercially, as long as the author is credited and the new creations are licensed under the identical terms.

For reprints contact: reprints@medknow.com

How to cite this article: Bulut DG, Kose E, Ozcan G, Sekerci AE, Canger EM, Sisman Y. Evaluation of root morphology and root canal configuration of premolars in the Turkish individuals using cone beam computed tomography. Eur J Dent 2015;9:551-7.

DOI: $10.4103 / 1305-7456.172624$ 
an ability to evaluate the maxillofacial anatomy in axial, sagittal, and coronal sections and produces high quality 3-D diagnostic images without structure overlapping. Root and canal morphology, the number of canals, and their divergence or convergence from each other can be visualized in 3-D. For these reasons, $\mathrm{CBCT}$ has been recommended for the accurate evaluation of root canal systems. ${ }^{[4]}$

In the literature, root canal morphology of upper premolar teeth has not been reported much more. Only it has specified that the canal morphologies are complex and changeable in maxillary upper premolar and the only tooth that shows all eight possible configurations of Vertucci's classification is maxillary second premolar. ${ }^{[1,5]}$ The morphology of mandibular premolars is very similar, and the root canal systems of these single-rooted premolars generally have a single root canal. However, reports have exhibited that the root canal morphology of premolars is not as easy as it may appear to be on plain radiographs. ${ }^{[2]}$ Many roots have additional canals and a variety of canal configurations. ${ }^{[5]}$

The aim of the present study was to analyze the accurate determination of root anatomy and morphology and root canal configuration of premolars in a Turkish population by using CBCT.

\section{MATERIALS AND METHODS}

The Ethical Committee of the University of Erciyes, Faculty of Dentistry, approved the study protocol that has, therefore, been performed according to the ethical standards laid down in the declaration of Helsinki in 1964.

We designed a retrospective study using CBCT images (NewTom 5G with a voxel size of $0.125 \mathrm{~mm}$ ) of maxillary and mandibular permanent premolars from patients who visited the Oral and Maxillofacial Radiology Department at the Erciyes University, Faculty of Dentistry, between June 2012 and March 2014. CBCT images investigated were taken because of dentomaxillofacial problems of patients.

We searched a database of 925 CBCT scans and evaluated 404 patients who met the following inclusion criteria:

- Permanent premolars with no periapical lesions

- Absence of root canal therapy

- No root canals with open apices

- Premolar teeth with complete root formation

- Absence of coronal or postcoronal restorations

- Absence of root resorption or calcification

- Presence of high-quality CBCT images.
The final group included records from 404 patients (199 females [49.3\%] and 205 males [50.7\%]). A total of 511 maxillary first premolar teeth, 476 second premolar teeth, 598 mandibular first premolar teeth, and 549 second premolar teeth were selected.

The CBCT images were examined in the NNT viewer which is a simple version of the NNT software of the CBCT (Newtom5G, QR, Verona, Italy) machine in a Dell Precision T5400 workstation (Dell, Round Rock, TX, USA), $0.25 \mathrm{~mm}$ isotropic voxel size, and a 32 inches Dell LCD screen with a resolution of $1,280 \times 1,024$ pixels in a darkroom. The brightness and contrast of the images were attuned using the image processing device in the software to ensure optimal image.

All the images were assessed concurrently by two dentomaxillofacial radiologists to reach an agreement in the interpretation of the radiographic findings. Types of canal configurations and the existence of a canal were investigated using the NNT toolbar. We rolled downward throughout the images from the bottom of the pulp chamber to the root tip to notice the number of canals and roots and the canal configuration at the axial slices in tomography. Tomography slices of $0.25 \mathrm{~mm}$ in the coronal, axial, and sagittal view were produced. Cross-sectional and axial views were sent out to a computer in the digital imaging and communications in medicine format. These images were used to inspect the root and root canal system.

Using CBCT, the teeth related were inspected for the following observations that estimated:

- The number and morphology of roots

- The number of canals in each root

- The canal morphology for each root according to Vertucci's classification ${ }^{[6]}$ [Figure 1].

For checking the diagnostic repeatability of the interreliability of the investigators, $10 \%$ of CBCT images chosen randomly by them were investigated each day for two consecutive weeks. Results were examined by the Wilcoxon matched pairs signed-rank test and it did not show any significant differences between the two observers.

The data examinations were performed by using the Statistical Package for the Social Sciences, version 16.0 (SPSS Inc., Chicago, IL, USA). The total numbers of roots, the root canal configuration, and unilateral or bilateral occurrences were analyzed. The incidence and the correlations among right and left side and between females and males were determined. The Chi-square test was used to evaluate the statistically 


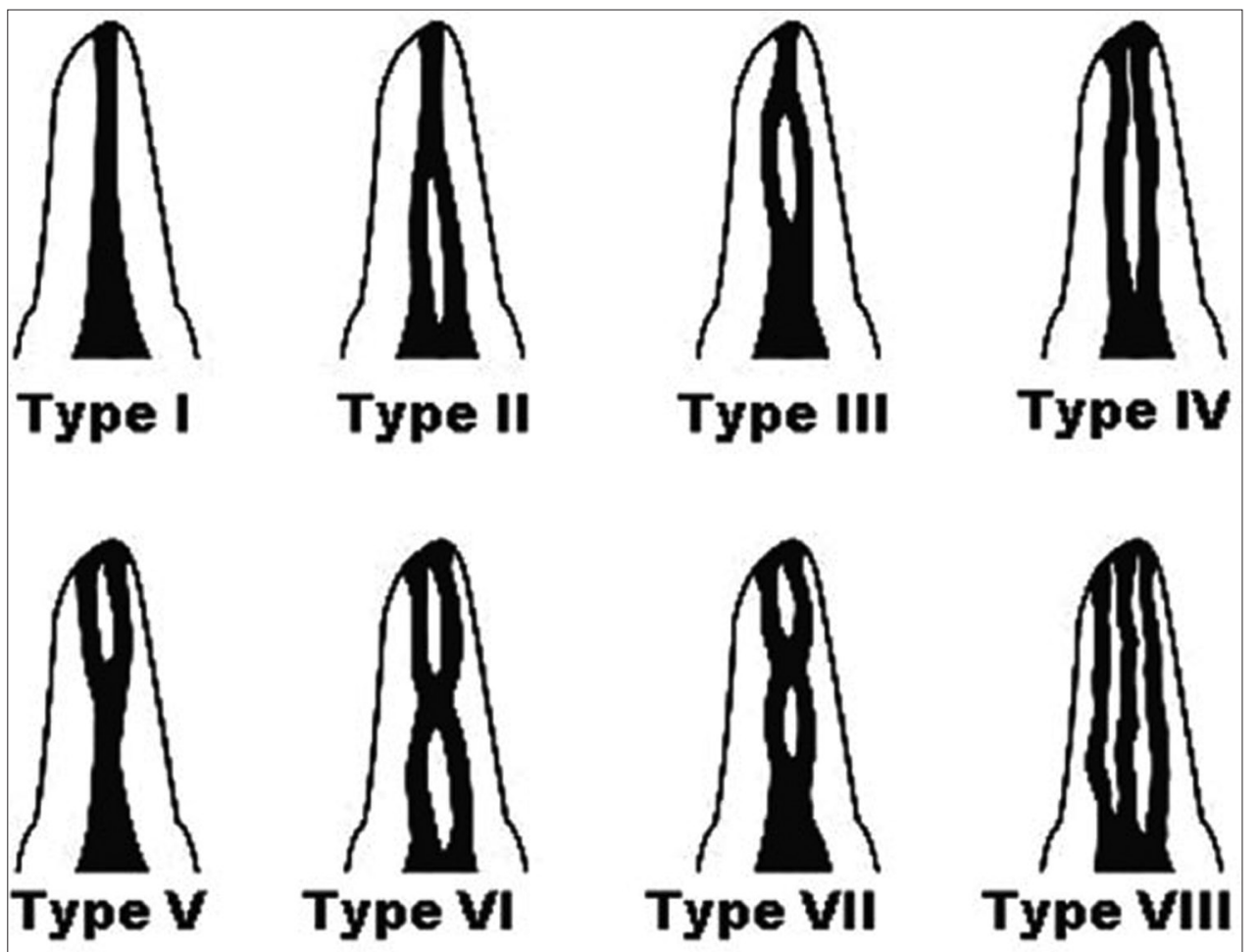

Figure 1: Classification of root canal system according to Vertucci (1984)

significant differences between both genders. Statistical significance was identified at the level of $P<0.05$.

\section{RESULTS}

Among the patients registered, 199 of them were female and 205 were male, with a mean age of 39.4 years (standard deviation: 16.67), varying from 15 to 77 years. In maxillary first premolar teeth, $41.4 \%$ of the patients had bilateral, $38.1 \%$ had unilateral premolar teeth, and $20.5 \%$ had no first premolar teeth, in second premolar; $38.8 \%$ of the patients had bilateral, $37.4 \%$ had unilateral premolar teeth, and $23.8 \%$ had no first premolar teeth. For the mandibular first premolar, $44.9 \%$ of the patients had bilateral, $42.4 \%$ had unilateral premolars, and $12.6 \%$ had no first premolar, in second premolar; $43.5 \%$ of the patients had bilateral, $39.3 \%$ had unilateral premolars, and $17.2 \%$ had no first premolar.

\section{Maxillary premolars}

The data for the frequency distribution of root morphology and root canal configuration are presented in Table 1. Variants in root canal morphology of the upper first and second premolars and the canal classifications were shown in Figure 2.

The majority $(70.8 \%)$ of maxillary first premolar teeth had two roots and one root canal in each root.

\begin{tabular}{|c|c|c|c|c|}
\hline \multirow[t]{2}{*}{ Number of roots } & \multicolumn{2}{|c|}{ Maxillary (\%) } & \multicolumn{2}{|c|}{ Mandibular (\%) } \\
\hline & $\begin{array}{c}\text { First } \\
\text { premolar }\end{array}$ & $\begin{array}{l}\text { Second } \\
\text { premolar }\end{array}$ & $\begin{array}{c}\text { First } \\
\text { premolar }\end{array}$ & $\begin{array}{l}\text { Second } \\
\text { premolar }\end{array}$ \\
\hline One root & 144 & 391 & 581 & 543 \\
\hline Two separate roots & 253 & 43 & 4 & 2 \\
\hline Two fused roots & 104 & 39 & 15 & 3 \\
\hline $\begin{array}{l}\text { Two apically } \\
\text { separate roots }\end{array}$ & 5 & 3 & 4 & 1 \\
\hline Three roots & 5 & 0 & 0 & 0 \\
\hline \multicolumn{5}{|l|}{$\begin{array}{l}\text { Number of canal } \\
\text { configuration }(\%)\end{array}$} \\
\hline Type 1 & $784(62.6)$ & $439(77.6)$ & $585(94.2)$ & $549(98.9)$ \\
\hline Type 2 & $427(34.1)$ & $71(12.5)$ & $4(0.64)$ & $1(0.2)$ \\
\hline Type 3 & $10(0.8)$ & $6(1.33)$ & $7(1,12)$ & $2(0.4)$ \\
\hline Type 4 & $24(1.9)$ & $37(6.5)$ & $5(0,80)$ & 0 \\
\hline Type 5 & $7(0.6)$ & $11(1.9)$ & $20(3.24)$ & $3(0.5)$ \\
\hline Type 6 & 0 & $1(0.17)$ & 0 & 0 \\
\hline Type 7 & 0 & 0 & 0 & 0 \\
\hline Type 8 & 0 & 0 & 0 & 0 \\
\hline
\end{tabular}

Otherwise, $28.2 \%$ of upper first premolars had one root and canal and $1 \%$ had three roots. All three-rooted first premolars were determined in male patients. While maxillary second premolars had usually one root and canal at a rate of $82.1 \%, 17.8 \%$ of them had two roots and canals [Table 1]. 

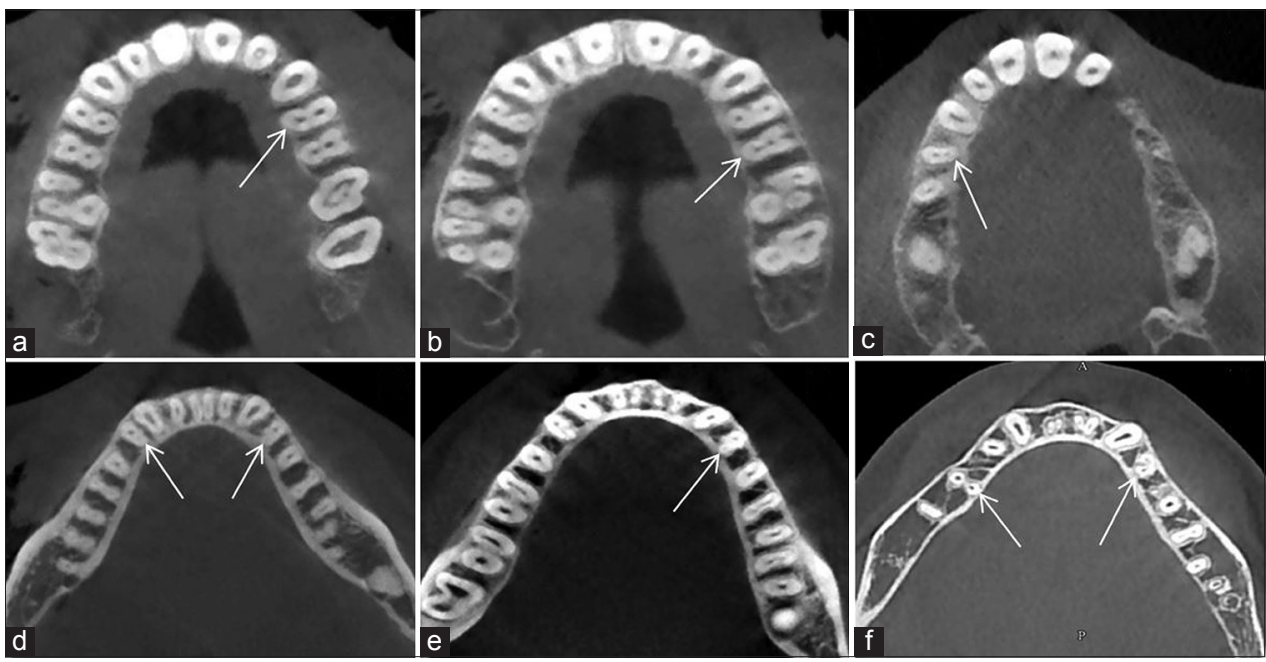

Figure 2: Root canal configuration of maxillary premolars (a) type I, (b) type II, (c) type IV, root canal configuration of mandibular premolars, (d) type III, (e) type V, and (f) type VI

Among two-rooted maxillary first premolars, the number of teeth with fused roots and apically separate roots were $104(28 \%)$ and $5(1,4 \%)$, respectively. Likewise, among two-rooted maxillary second premolars, the number of teeth with fused roots and apically separate roots were $39(45 \%)$ and $3(3,5 \%)$, respectively [Figure 3].

The numbers of roots were not revealed the difference in females and males except right maxillary second premolar [Table 2]. All three-rooted premolars were seen in males. When the maxillary first and second premolars were evaluated unilaterally, the incidence of canals was not diverse between the males and females. The frequency distribution of the different canal configurations of the maxillary premolars is shown in Table 1. In maxillary premolars, the type I canal configuration was the most prevalent in first premolars in proportion to $62,6 \%, 77,6 \%$ of second premolars, irrespective of the gender. The type VII and VIII canal configuration was not found in all teeth. For the maxillary premolars, when the teeth were evaluated unilaterally, there were no variations in the root canal configurations and the numbers of canals between the left and right side of both females and males.

\section{Mandibular premolars}

Table 1 shows the frequency distribution of the different canal configurations of the lower premolar teeth. Overall, in lower first premolar teeth type I, which indicated one root canal, was the most prevalent with the rate of $94.2 \%(n=585)$ and $0.6 \%(n=4)$ of the teeth had type II canal configuration. The type III canal configuration was observed in $1 \%(n=7)$ of cases

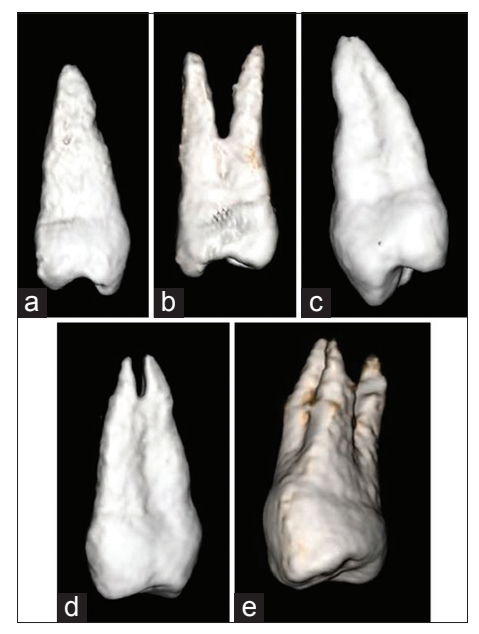

Figure 3: Root morphology of premolars (a) one-rooted, (b) two separate roots, (c) two-fused root, (d) two apically separate roots, and (e) three separate roots

followed by type IV in $0.8 \%(n=5)$ and type IV in $3.2 \%$ $(n=20)$. Type VII and VIII canal configurations were not found in all teeth. For the incidences of varying root canal configurations, there was no difference between the left and the right side of both males and females.

In the mandibular second premolar teeth, type I canal configuration was the most prevalent ( $n=549,98.9 \%$ ) and only one teeth had type II canal configuration. The type $\mathrm{V}$ canal configuration was observed in three cases. Type IV, VII, and VIII canal configurations were not found in all teeth. Regardless of sex, for the incidences of varying root canal configurations, there was no difference between the left and the right side $(P>0.05)$. The numbers of roots were not revealed the difference in females and males except 


\begin{tabular}{|c|c|c|c|c|c|c|}
\hline & \multicolumn{5}{|c|}{ Number of roots } & \multirow[t]{3}{*}{$P$} \\
\hline & \multicolumn{3}{|c|}{ Male } & \multicolumn{2}{|c|}{ Female } & \\
\hline & $\begin{array}{l}\text { One } \\
\text { root }\end{array}$ & $\begin{array}{l}\text { Two } \\
\text { roots }\end{array}$ & $\begin{array}{l}\text { Three } \\
\text { roots }\end{array}$ & $\begin{array}{l}\text { One } \\
\text { root }\end{array}$ & $\begin{array}{l}\text { Two } \\
\text { roots }\end{array}$ & \\
\hline \multicolumn{7}{|l|}{ Maxillary } \\
\hline Right first premolar & 29 & 97 & 2 & 36 & 93 & 0.242 \\
\hline Right second premolar & 92 & 30 & & 105 & 16 & $0.024^{*}$ \\
\hline Left first premolar & 39 & 90 & 3 & 40 & 82 & 0.224 \\
\hline Left second premolar & 92 & 23 & & 102 & 16 & 0.188 \\
\hline \multicolumn{7}{|l|}{ Mandibular } \\
\hline Right first premolar & 152 & 7 & & 137 & 4 & 0.471 \\
\hline Right second premolar & 142 & 1 & & 120 & 1 & 0.906 \\
\hline Left first premolar & 142 & 8 & & 144 & 4 & 0.248 \\
\hline Left second premolar & 145 & 4 & & 136 & 0 & $0.004^{*}$ \\
\hline
\end{tabular}

left mandibular second premolar and right maxillary second premolar $(P=0.004, P=0.024$ respectively $)$. All two-rooted premolars were seen in males [Table 2].

\section{DISCUSSION}

This study provides a report on the root and canal morphology of maxillary and mandibular premolars in a Turkish population by using CBCT. It is important to have a thorough knowledge of the root anatomy and canal structure for a successful root canal therapy. Numerous methods have been used for understanding the internal morphology of roots and canals. ${ }^{[7]}$ Canal configuration has been assessed by tooth clearing and canal staining, ${ }^{[8]}$ in vitro macroscopic examination, ${ }^{[9]}$ in vivo root canal treatment with magnification, ${ }^{10]}$ plastic resin injection, ${ }^{[11]}$ sectioning ${ }^{[12]}$ scanning electron microscopy evaluation, ${ }^{[13]}$ contrast medium-enhanced radiographic techniques, ${ }^{[14]}$ conventional radiographs, ${ }^{[15]}$ computed tomography (CT), micro-CT, and CBCT. ${ }^{[16]}$ Differences in study design and the various origins of the inspected teeth could account for highly variable outcomes. ${ }^{[7]}$

Today, CBCT imaging system produces higher resolution volumetric records, high lower scan time, and geometric accuracy with a low radiation dose. ${ }^{[17]}$ Innovations in imaging systems and increased usage of CBCT in dentistry have allowed us to have a more accurate and closer look at anatomical structures. This technique has the potential to replace CT scans for correct diagnosis and evaluation of the internal and external morphology of the root and canal system. The use of CBCT after the 2000s to provide 3-D imaging, due to lower radiation dose and lower costs compared to CTs, has been accepted as a valuable imaging technique by many dentomaxillofacial authorities. In accordance with the present study, the morphology of roots and canals can be determined visibly in axial sections. ${ }^{[16]}$

The anatomy of root canals was categorized into eight types according to Vertucci classification, and we used this classification in the present study. ${ }^{[18]}$ Many studies have reported that variations in root canal anatomy are very common. ${ }^{[19]}$ In some studies, the numbers of root canals of maxillary first premolars were reported the percentage; one as $3.92-26.2 \%$, two as $73.3-97 \%$, and three as $0-5 \% .{ }^{[14,20-22]}$ In the study by Ok et al., the percentage of maxillary first premolars which had one root and one canal was $12.5 \%$. In the present study, $28.2 \%$ of upper first premolar had one root and one canal which is higher than previous studies and the majority $(70.8 \%)$ of upper first premolar had two roots and two canals that is slightly lower than previous studies. ${ }^{[20,21]}$ The percentage of three-canalled $(1 \%)$ maxillary first premolars was higher than Caliskan et al., ${ }^{[20]}$ same with Ok et al. $(1 \%)^{[18]}$ and lower than Kartal et al. $(1.6 \%){ }^{[21]}$

Several studies and textbooks represent Vertucci's type I classification as the most common finding among all the types. ${ }^{[22-26]}$ In the previous studies of the Turkish population, ${ }^{[18,20,21,27]}$ it was reported that the type IV canal configuration was the most common with a percentage of $60-78 \%$ in the maxillary first premolar teeth. However, in the present study, type 1 was the most common canal configuration with a ratio of $62,6 \%$; same as the results of many studies and textbooks.

In other previous studies, related of maxillary second premolars, were reported that the $44-55 \%$ of teeth was one-canalled, $45-56 \%$ was two-canalled, and $0-1 \%$ was three-canalled. ${ }^{[14,20,21,28]}$ In the present study, maxillary second premolars had usually one root and canal $(82.1 \%)$ that was higher than found in several studies of the Turkish population. ${ }^{[20,21]} 17.8 \%$ were having two roots and canals. When compared with the earlier studies of a Turkish population, three roots and canals could not observed in this study. ${ }^{[20,21]}$

In the literature, the incidence of one-canalled mandibular first premolars and second premolars were reported as $74-89.5 \%$ and $81.5-98.8 \%$, respectively, similar to the results of present study. ${ }^{[14,20,27,29-31]}$ Also in the current study, when the mandibular first and 
second premolars were evaluated, sex predilection was not observed. For the incidences of varying root canal configurations, there was no difference between the left and the right side.

In the present study, the type I canal configuration was the most common in mandibular second premolar with a quite high ratio at $98.9 \%$. In previous in vitro studies of the Turkish population, ${ }^{[20,21,27,29]}$ the prevalence of type I configuration for mandibular premolars were found less than our results but in the study of E. Ok et al. was same as ours, approximately. The discrepancy in premolars in the current study compared to these studies may be related to most factors, with ethnic origins ${ }^{[32]}$ and ethnic differences, ${ }^{[19]}$ the major reasons for the diversity in the canal configurations. The previous studies of a Turkish population were performed in the North-west and Western regions of Turkey. In contrast, the CBCT data in the present in vivo study were obtained from the patients living in the Cappadocia region of the country. We could say that ethnic discrepancy between populations may influence the morphology of the root canals. These results emphasize the need for a careful radiographic examination in the success of root canal therapy.

\section{CONCLUSIONS}

The present study has some different results compared with the other study ${ }^{[18]}$ of the Turkish population, especially for Vertucci type of maxillary first premolars. This study provides comprehensive information for dentists on the root canal morphology of maxillary and mandibular premolar teeth in a Turkish population. CBCT was a clinically useful tool which leads to favorable endodontic treatment.

\section{Financial support and sponsorship}

Nil.

\section{Conflicts of interest}

There are no conflicts of interest.

\section{REFERENCES}

1. Hargreoues KM, Cohen S. Cohen's Pathways of the Pulp. $10^{\text {th }}$ ed. China: Mosby Elsevier Inc.; 2011. p. 141-7.

2. Lin Z, Hu Q, Wang T, Ge J, Liu S, Zhu M, et al. Use of CBCT to investigate the root canal morphology of mandibular incisors. Surg Radiol Anat 2014;36:877-82

3. Patel S, Dawood A, Ford TP, Whaites E. The potential applications of cone beam computed tomography in the management of endodontic problems. Int Endod J 2007;40:818-30.

4. Tian YY, Guo B, Zhang R, Yu X, Wang H, Hu T, et al. Root and canal morphology of maxillary first premolars in a Chinese subpopulation evaluated using cone-beam computed tomography. Int Endod J 2012;45:996-1003.

5. Vertucci FJ. Root canal morphology and its relationship to endodontic procedures. Endod Topics 2005;10:3-29.

6. Vertucci FJ, Williams RG. Root canal anatomy of the mandibular first molar. J N J Dent Assoc 1974;45:27-8.

7. Nur BG, Ok E, Altunsoy M, Aglarci OS, Colak M, Gungor E. Evaluation of the root and canal morphology of mandibular permanent molars in a south-eastern Turkish population using cone-beam computed tomography. Eur J Dent 2014;8:154-9.

8. Gulabivala K, Opasanon A, Ng YL, Alavi A. Root and canal morphology of Thai mandibular molars. Int Endod J 2002;35:56-62.

9. Pécora JD, Woelfel JB, Sousa Neto MD. Morphologic study of the maxillary molars 1. External anatomy. Braz Dent J 1991;2:45-50.

10. Buhrley LJ, Barrows MJ, BeGole EA, Wenckus CS. Effect of magnification on locating the MB2 canal in maxillary molars. J Endod 2002;28:324-7.

11. Skidmore AE, Bjorndal AM. Root canal morphology of the human mandibular first molar. Oral Surg Oral Med Oral Pathol 1971;32:778-84.

12. Seidberg BH, Altman M, Guttuso J, Suson M. Frequency of two mesiobuccal root canals in maxillary permanent first molars. J Am Dent Assoc 1973;87:852-6.

13. Sperber GH, Moreau JL. Study of the number of roots and canals in Senegalese first permanent mandibular molars. Int Endod J 1998;31:117-22.

14. Fan B, Gao Y, Fan W, Gutmann JL. Identification of a C-shaped canal system in mandibular second molars-part II: The effect of bone image superimposition and intraradicular contrast medium on radiograph interpretation. J Endod 2008;34:160-5.

15. Pineda F, Kuttler Y. Mesiodistal and buccolingual roentgenographic investigation of 7,275 root canals. Oral Surg Oral Med Oral Pathol 1972;33:101-10.

16. Demirbuga S, Sekerci AE, Dinçer AN, Cayabatmaz M, Zorba YO. Use of cone-beam computed tomography to evaluate root and canal morphology of mandibular first and second molars in Turkish individuals. Med Oral Patol Oral Cir Bucal 2013;18:e737-44.

17. Altunsoy M, Ok E, Nur BG, Aglarci OS, Gungor E, Colak M. A cone-beam computed tomography study of the root canal morphology of anterior teeth in a Turkish population. Eur J Dent 2014;8:302-6.

18. Ok E, Altunsoy M, Nur BG, Aglarci OS, Çolak M, Güngör E. A cone-beam computed tomography study of root canal morphology of maxillary and mandibular premolars in a Turkish population. Acta Odontol Scand 2014;72:701-6.

19. Awawdeh L, Abdullah H, Al-Qudah A. Root form and canal morphology of Jordanian maxillary first premolars. J Endod 2008;34:956-61.

20. Caliskan MK, Pehlivan Y, Sepetçioglu F, Türkün M, Tuncer SS. Root canal morphology of human permanent teeth in a Turkish population. J Endod 1995;21:200-4.

21. Kartal N, Ozçelik B, Cimilli H. Root canal morphology of maxillary premolars. J Endod 1998;24:417-9.

22. Vertucci FJ, Gegauff A. Root canal morphology of the maxillary first premolar. J Am Dent Assoc 1979;99:194-8.

23. Vertucci FJ. Root canal anatomy of the human permanent teeth. Oral Surg Oral Med Oral Pathol 1984;58:589-99.

24. Slowey RR. Root canal anatomy. Road map to successful endodontics. Dent Clin North Am 1979;23:555-73.

25. England MC Jr, Hartwell GR, Lance JR. Detection and treatment of multiple canals in mandibular premolars. J Endod 1991;17:174-8.

26. Shetty A, Hegde MN, Tahiliani D, Shetty H, Bhat GT, Shetty S. A three-dimensional study of variations in root canal morphology using cone-beam computed tomography of mandibular premolars in a South Indian population. J Clin Diagn Res 2014;8:ZC22-4.

27. Sert S, Bayirli GS. Evaluation of the root canal configurations of the mandibular and maxillary permanent teeth by gender in the Turkish population. J Endod 2004;30:391-8.

28. Vertucci F, Seelig A, Gillis R. Root canal morphology of the human maxillary second premolar. Oral Surg Oral Med Oral Pathol 1974;38:456-64.

29. Sert S, Aslanalp V, Tanalp J. Investigation of the root canal 
configurations of mandibular permanent teeth in the Turkish population. Int Endod J 2004;37:494-9.

30. Vertucci FJ. Root canal morphology of mandibular premolars. J Am Dent Assoc 1978;97:47-50.

31. Llena C, Fernandez J, Ortolani PS, Forner L. Cone-beam computed tomography analysis of root and canal morphology of mandibular premolars in a Spanish population. Imaging Sci Dent 2014;44:221-7.

32. Trope M, Elfenbein L, Tronstad L. Mandibular premolars with more than one root canal in different race groups. J Endod 1986;12:343-5.

\begin{tabular}{|l|l|}
\hline \multicolumn{2}{|c|}{ Access this article online } \\
\hline Quick Response Code: \\
\hline
\end{tabular}

